\title{
WAVE FRONTS OF SOLUTIONS OF SOME CLASSES OF NON-LINEAR PARTIAL DIFFERENTIAL EQUATIONS
}

\author{
P. R. POPIVANOV \\ Department of Mathematics, Sofia University \\ Bul. A. Ivanov 5, Sofia, Bulgaria
}

1. This paper is devoted to the study of wave fronts of solutions of first order symmetric systems of non-linear partial differential equations. A short communication was published in [4]. The microlocal point of view enables us to obtain more precise information concerning the smoothness of solutions of symmetric hyperbolic systems. Our main result is a generalization to the non-linear case of Theorem 1.1 of Ivriu [3]. The machinery of paradifferential operators introduced by Bony [1] together with an idea coming from [3], [2] are used.

2. The definition and main properties of paradifferential operators are assumed to be known to the reader [1]. We will use here the same notations as in [1]. We recall the definition of the microlocalized Sobolev space $\mathcal{H}_{\text {mcl }}^{s}$ :

Definition. A distribution $u \in \mathcal{D}^{\prime}(X)$ belongs to the class $\mathcal{H}_{\text {mcl }}^{s}\left(\varrho^{0}\right), \varrho^{0} \in$ $T^{*}(X) \backslash 0, \varrho^{0}=\left(x^{0}, \xi^{0}\right)$, if there exists a classical properly supported pseudodifferential operator $a$ of order 0 such that $a\left(\varrho^{0}\right) \neq 0$, au $\in \mathcal{H}_{\text {loc }}^{s}(X)$, where $\mathcal{H}_{\text {loc }}^{s}$ is the local Sobolev space.

We denote by $W \subset T^{*}(X) \backslash 0$ an (open) closed set conical with respect to $\xi$ and having a compact base in $X$. Assume that $F_{k}\left(x, u_{1}, \ldots, u_{N}, u_{11}, \ldots, u_{i j}, \ldots\right.$, $\left.u_{N n}\right), 1 \leq j \leq n, 1 \leq i, k \leq N$, are real-valued $C^{\infty}$-functions of their arguments $x \in X, \vec{u} \in \mathbb{R}^{N},\left(u_{11}, \ldots, u_{N n}\right) \in \mathbb{R}^{N n}$ and $X$ is a domain in $\mathbb{R}^{n}$. Define a matrix $A_{j}$ by

$$
A_{j}=\left\|\partial F_{k} / \partial u_{i j}(x, \vec{u}(x), \partial \vec{u}(x))\right\|_{1 \leq i, k \leq N} .
$$

We now formulate the main result of this paper. 
THEOREM 1. Consider the non-linear system of partial differential operators

$$
F_{k}(x, \vec{u}(x), \partial \vec{u}(x))=0, \quad 1 \leq k \leq N,
$$

$\vec{u}(x)=\left(u_{1}, \ldots, u_{N}\right)$, and suppose that (1) possesses a real-valued solution $\vec{u} \in$ $\mathcal{H}_{\text {loc }}^{s}(X), s>2+n / 2$, such that

(i) $\partial F_{k} / \partial u_{i j}(x, \vec{u}(x), \partial \vec{u}(x))=\partial F_{i} / \partial u_{k j}(x, \vec{u}(x), \partial \vec{u}(x)), \quad \forall x \in X$,

(ii) the matrix $A_{j_{0}}(x)=\left\|\partial F_{k} / \partial u_{i j_{0}}(x, \vec{u}(x), \partial \vec{u}(x))\right\|_{1 \leq i, k \leq N}, x \in X$, is (positive) negative definite.

Suppose, moreover, that for each characteristic point $\varrho^{0} \in$ Char $p_{1} \cap \partial W \cap$ $\left\{x_{j_{0}} \geq \delta\right\}$ we have $u \in \mathcal{H}_{\mathrm{mcl}}^{t}\left(\varrho^{0}\right)$ for some $t<2 s-2-n / 2$. Then $u \in \mathcal{H}_{\mathrm{mcl}}^{t}\left(\varrho^{0}\right)$, $\forall \varrho^{0} \in$ Char $p_{1} \cap W \cap\left\{x_{j_{0}} \geq \delta\right\}, \delta=$ const.

We point out that conditions (i), (ii) imply that the linearized system $P v=$ $\sum_{j=1}^{n} A_{j}(x) D_{j} v-i B(x) v$ is symmetric and positive, $B, A_{j}(x) \in C^{1+\varepsilon}(X), 1>$ $\varepsilon>0$. As usual,

$$
\text { Char } p_{1}=\left\{\varrho=(x, \xi) \in T^{*}(X) \backslash 0: \operatorname{det} \sum_{j=1}^{n} A_{j}(x) \xi_{j}=0\right\} .
$$

It is interesting to note that $u \in \mathcal{H}_{\mathrm{mcl}}^{2 s-1-\varepsilon-n / 2}\left(\varrho^{0}\right), \varepsilon>0$, for each $\varrho^{0} \notin$ Char $p_{1}$ (see Th. 5.4 of $[1]$ ).

Standard considerations from the theory of paradifferential operators $P \in$ $\widetilde{O}_{p}\left(\Sigma_{\sigma}^{1}\right), \sigma>1, \sigma$ not an integer, reduce the proof of Theorem 1 to the proof of the following assertion.

TheOREM 2. Consider the first order paradifferential system

$$
P(x, D) u=\sum_{j=1}^{n} A_{j}(x) D_{j} u-i B(x) u=f \quad(-P(x, D) u=-f)
$$

where $P \in \widetilde{O}_{p}\left(\Sigma_{\sigma}^{1}\right), \sigma>1, \sigma$ not an integer, $A_{j}^{*}(x)=A_{j}(x), \forall x \in X$, the $A_{j}(x)$ are real-valued $N \times N$ matrices and $\left(A_{j_{0}}(x)>0\right) A_{j_{0}}(x)<0, \forall x \in X$. Assume that $u \in \mathcal{H}_{\mathrm{comp}}^{t-1 / 2}(X), P u \in \mathcal{H}_{\mathrm{mcl}}^{t}\left(W \cap\left\{x_{j_{0}} \geq \delta\right\}\right)$, and $u \in \mathcal{H}_{\mathrm{mcl}}^{t}\left(\varrho^{0}\right)$ for each $\varrho^{0} \in \operatorname{Char} p_{1} \cap \partial W \cap\left\{x_{j_{0}} \geq \delta\right\}$. Then

$$
u \in \mathcal{H}_{\text {mcl }}^{t}\left(\varrho^{0}\right), \quad \forall \varrho^{0} \in \operatorname{Char} p_{1} \cap W \cap\left\{x_{j_{0}} \geq \delta\right\} .
$$

In the special case when $\varrho^{0} \notin$ Char $p_{1}$ the solution $u \in \mathcal{H}_{\mathrm{mcl}}^{t+1}\left(\varrho^{0}\right)$.

3. Supposing Theorem 2 is proved and $s<t$ we will verify Theorem 1 . To do this we apply Theorem $5.3 \mathrm{~b}$ ) of [1] with the corresponding notations $d=1$, $\varrho=s-\varepsilon-n / 2, \varepsilon>0, \sigma=\varrho-1$ to conclude that there exists a paradifferential operator $P \in \widetilde{O}_{p}\left(\Sigma_{\sigma}^{1}\right), \sigma>1$, satisfying $P u \in \mathcal{H}_{\mathrm{loc}}^{2 s-2-\varepsilon-n / 2} \Rightarrow P u \in \mathcal{H}_{\mathrm{loc}}^{t}$ for $\varepsilon>0$ sufficiently small, $u \in \mathcal{H}_{\text {loc }}^{s}$. 
The next remark will be useful later:

Let $u \in \mathcal{H}_{\mathrm{loc}}^{s}(X), P u \in \mathcal{H}_{\mathrm{mcl}}^{t}\left(W \cap\left\{x_{j_{0}} \geq \delta\right\}\right), u \in \mathcal{H}_{\mathrm{mcl}}^{t-1 / 2}\left(W \cap\left\{x_{j_{0}} \geq \delta\right\}\right)$ and $u \in \mathcal{H}_{\mathrm{mcl}}^{t}\left(\partial W \cap\left\{x_{j_{0}} \geq \delta\right\}\right)$. Then $u \in \mathcal{H}_{\text {mcl }}^{t}\left(W \cap\left\{x_{j_{0}} \geq \delta\right\}\right)$.

In fact, consider a classical pseudodifferential operator $T \in S_{1,0}^{0}, T \equiv 1$ in a small conic neighbourhood (ngbhd) of $W \cap\left\{x_{j_{0}} \geq \delta\right\}, T \equiv 0$ outside a larger conic ngbhd of $W \cap\left\{x_{j_{0}} \geq \delta\right\}$. Then $T u \in \mathcal{H}_{\text {comp }}^{t-1 / 2}(X), T u \in \mathcal{H}_{\text {mcl }}^{t}\left(\partial W \cap\left\{x_{j_{0}} \geq\right.\right.$ $\delta\})$ and $P(T u) \in \mathcal{H}_{\mathrm{mcl}}^{t}\left(W \cap\left\{x_{j_{0}} \geq \delta\right\}\right)$ as $P(T u)=P u+P((T-I) u)$ and $P((I-T) u) \in \mathcal{H}_{\mathrm{mcl}}^{s-1+\sigma} \subset \mathcal{H}_{\mathrm{mcl}}^{t}\left(W \cap\left\{x_{j_{0}} \geq \delta\right\}\right)$ according to Corollary 3.5 of [1]. Thus $T u \in \mathcal{H}_{\text {mcl }}^{t}\left(W \cap\left\{x_{j_{0}} \geq \delta\right\}\right) \Rightarrow u \in \mathcal{H}_{\text {mcl }}^{t}\left(W \cap\left\{x_{j_{0}} \geq \delta\right\}\right)$. To complete the proof of Theorem 1 we observe that there exists a uniquely determined integer $k \geq 1$ for which $(k-1) / 2 \leq t-s<k / 2$ and therefore

$$
t-k / 2 \leq s \leq t-(k-1) / 2<t-(k-2) / 2<\ldots<t-1 / 2<t .
$$

Setting $t^{\prime}=t-(k-1) / 2$ we get $u \in \mathcal{H}_{\mathrm{loc}}^{s} \subset \mathcal{H}_{\mathrm{loc}}^{t-k / 2}=\mathcal{H}_{\mathrm{loc}}^{t^{\prime}-1 / 2}, P u \in \mathcal{H}_{\mathrm{loc}}^{t} \subset$ $\mathcal{H}_{\mathrm{loc}}^{t-(k-1) / 2}=\mathcal{H}_{\mathrm{loc}}^{t^{\prime}}, u \in \mathcal{H}_{\mathrm{mcl}}^{t^{\prime}}\left(\partial W \cap\left\{x_{j_{0}} \geq \delta\right\}\right)$. So $u \in \mathcal{H}_{\mathrm{mcl}}^{t^{\prime}}\left(W \cap\left\{x_{j_{0}} \geq \delta\right\}\right)$ as $s \leq t^{\prime}$. Put now $t^{\prime \prime}=t-(k-2) / 2=t^{\prime}+1 / 2$. Obviously $u \in \mathcal{H}_{\mathrm{mcl}}^{t^{\prime \prime}-1 / 2}\left(W \cap\left\{x_{j_{0}} \geq\right.\right.$ $\delta\}), P u \in \mathcal{H}_{\text {loc }}^{t^{\prime \prime}}, u \in \mathcal{H}_{\text {mcl }}^{t^{\prime \prime}}\left(\partial W \cap\left\{x_{j_{0}} \geq \delta\right\}\right)$.

The remark above and $s \leq t^{\prime} \leq t^{\prime \prime}$ give us $u \in \mathcal{H}_{\text {mcl }}^{t^{\prime \prime}}\left(W \cap\left\{x_{j_{0}} \geq \delta\right\}\right)$. Thus we conclude that $u \in \mathcal{H}_{\text {mcl }}^{t}\left(W \cap\left\{x_{j_{0}} \geq \delta\right\}\right)$.

4. Proof of Theorem 2. To simplify the proof we will assume that $W=$ $\Delta \times \Gamma_{\xi}, \Delta=\left[a_{1}, b_{1}\right] \times \ldots \times\left[a_{n}, b_{n}\right], \Gamma_{\xi}$ is a closed cone in $T^{*}\left(\mathbb{R}^{n}\right)$ and $A_{1}(x)<0$. Choose $\kappa_{j} \in C_{0}^{\infty}(\mathbb{R})$ so that $\kappa_{j} \equiv 1$ on $\left[a_{j}, b_{j}\right], \kappa_{j}^{\prime}\left(x_{j}\right)=\kappa_{j}^{-}\left(x_{j}\right)-\kappa_{j}^{+}\left(x_{j}\right), 0 \leq \kappa_{j}^{+}$, $0 \leq \kappa_{j}^{-}, x_{j} \leq a_{j}$ in $\operatorname{supp} \kappa_{j}^{-}, x_{j} \geq b_{j}$ in $\operatorname{supp} \kappa_{j}^{+}$and $\delta=a_{1}$ but no information on the $\mathcal{H}_{\mathrm{mcl}}^{t}$-smoothness of $u$ at $\left\{x_{1}=a_{1}\right\} \times \Gamma_{\xi}$ is given. For $\lambda, \delta_{1}>0$ put

$$
Q=Q_{\lambda, \delta_{1}}=e^{\lambda x_{1}} \kappa(x)\left(1+\left|\delta_{1} \xi\right|^{2}\right)^{-1} h(\xi),
$$

$\operatorname{ord}_{\xi} h=t$ and conesupp $Q_{\lambda, \delta_{1}}$ is concentrated in a small conic ngbhd of $W$. Obviously, $Q_{\lambda, \delta_{1}} \in S_{1,0}^{t-2}$ and the factor $\kappa(x)\left(1+\left(\delta_{1}|\xi|\right)^{2}\right)^{-1}$ is bounded in $\Sigma_{\varrho}^{0}$, $S_{1,0}^{0}, \forall \varrho>0$, $\varrho$ not an integer, uniformly with respect to $\delta_{1} \in(0,1]$ and $\kappa(x)=$ $\kappa_{1}(x) \ldots \kappa_{n}(x)$. Thus for each fixed $\lambda>0$ and arbitrary $\delta_{1} \in(0,1], Q_{\lambda, \delta_{1}} \in S_{1,0}^{t}$.

Consider now the identity

$$
(Q P u, Q u)_{L_{2}}=(P Q u, Q u)_{L_{2}}+([Q, P] u, Q u)_{L_{2}}
$$

It is legitimate as $P u \in \mathcal{H}_{\text {mcl }}^{t}(W) \Rightarrow Q P u \in \mathcal{H}_{\text {comp }}^{2}(X), Q u \in \mathcal{H}_{\text {comp }}^{3 / 2}(X)$ (in our notations $\left.W=W \cap\left\{x_{1} \geq \delta\right\}\right)$. So

$$
\operatorname{Im}(Q P u, Q u)_{L_{2}}=\operatorname{Im}(P Q u, Q u)_{L_{2}}+\operatorname{Im}([Q, P] u, Q u)_{L_{2}} .
$$

We first estimate

$$
I=\operatorname{Im}(P Q u, Q u)_{L_{2}},
$$


i.e. we have to consider the terms $\left(A_{j}(x) D_{j} Q u, Q u\right),(B(x) Q u, Q u)\left((\cdot, \cdot)_{L_{2}}=\right.$ $(\cdot, \cdot))$. It can easily be seen that

$$
|(B(x) Q u, Q u)| \leq C_{1}\|Q u\|_{0}^{2}+C_{1 \lambda}\|u\|_{t-\sigma / 2}^{2}
$$

where $C_{1}$ is an absolute constant and $C_{1 \lambda}$ depends on $\lambda>0$ but does not depend on $\delta_{1} \in(0,1]$. Now,

$$
\begin{aligned}
\left(A_{j}(x) D_{j} Q u, Q u\right) & =\left(D_{j} A_{j} Q u, Q u\right)+\left(\left[A_{j}, D_{j}\right] Q u, Q u\right) \\
& =\left(Q u, A_{j} D_{j} Q u\right)+\left(\left[A_{j}, D_{j}\right] Q u, Q u\right),
\end{aligned}
$$

i.e. $2\left|\operatorname{Im}\left(A_{j} D_{j} Q u, Q u\right)\right| \leq\left|\left(\left[A_{j}, D_{j}\right] Q u, Q u\right)\right|$. The principal symbol of the commutator $\left[A_{j}, D_{j}\right]$ is $-i\left\{A_{j}, \xi_{j}\right\}=i \partial A_{j}(x) / \partial \xi_{j} \in \Sigma_{\sigma-1}^{0}, \sigma-1>0$, i.e. $\operatorname{Im}\left|\left(A_{j} D_{j} Q u, Q u\right)\right| \leq C_{2}\|Q u\|_{0}^{2}$. In other words,

$$
|I| \leq C_{3}\|Q u\|_{0}^{2}+C_{3 \lambda}\|u\|_{t-\sigma / 2}^{2} .
$$

To estimate $I I=\operatorname{Im}([Q, P] u, Q u)$ we use Theorem 3.2 of [1]. Since the principal symbol of $[Q, P]$ is $(1 / i)\left\{Q, p_{1}\right\}$ we have

$$
I I=-\operatorname{Re}\left(\left\{Q, p_{1}\right\} u, Q u\right)+C_{3 \lambda}^{\prime}\|u\|_{t+(1-\sigma) / 2}^{2}
$$

Obviously

$$
-\left\{Q, p_{1}\right\}=-\sum_{j, k=1}^{n}\left(\partial Q / \partial \xi_{k}\right)\left(\partial A_{j}(x) / \partial x_{k}\right) \xi_{j}+\sum_{j=1}^{n}\left(\partial Q / \partial x_{j}\right) A_{j}(x)
$$

and therefore

$$
\partial Q / \partial x_{1}=\lambda Q+e^{\lambda x_{1}}\left(\partial \kappa / \partial x_{1}\right)\left(1+\left|\delta_{1} \xi\right|^{2}\right)^{-1} h(\xi) .
$$

The inequality $\partial \kappa / \partial x_{1} \geq-\kappa_{1}^{+}\left(x_{1}\right) \kappa_{2} \ldots \kappa_{n}=-\kappa^{+}(x)$ will enable us to apply the sharp Gårding estimate. In fact,

$$
\operatorname{Re}\left(\left(\partial Q / \partial x_{1}\right) A_{1} u, Q u\right)=\lambda \operatorname{Re}\left(Q A_{1} u, Q u\right)+\operatorname{Re}\left(\widetilde{Q}^{+} A_{1} u, Q u\right),
$$

where $\widetilde{Q}^{+}=e^{\lambda x_{1}}\left(\partial \kappa / \partial x_{1}\right)\left(1+\left|\delta_{1} \xi\right|^{2}\right)^{-1} h(\xi)$. It is clear that $\left(Q A_{1} u, Q u\right)=$ $\left(A_{1} Q u, Q u\right)+\left(\left[Q, A_{1}\right] u, Q u\right)$, thus

$$
\operatorname{Re}\left(Q A_{1} u, Q u\right) \leq-C_{4}\|Q u\|_{0}^{2}+C_{4 \lambda}\|u\|_{t-1 / 2}^{2}, \quad C_{4}>0 .
$$

On the other hand,

$$
\operatorname{Re}\left(\widetilde{Q}^{+} A_{1} u, Q u\right) \leq \operatorname{Re}\left(A_{1}(x) \kappa\left(\partial \kappa / \partial x_{1}\right) v, v\right)+C_{5 \lambda}\|u\|_{t-1 / 2}^{2}
$$

where $v=e^{\lambda x_{1}} h(D)\left(1+\left|\delta_{1} D\right|^{2}\right)^{-1} u$. The commutator

$$
\left[A_{1}, \kappa e^{\lambda x_{1}}\left(\partial \kappa / \partial x_{1}\right) h(D)\left(1+\left|\delta_{1} D\right|^{2}\right)^{-1}\right]
$$

is bounded in $\Sigma_{\sigma-1}^{t-1}$ uniformly with respect to $\delta_{1}>0$. We apply the sharp Gårding inequality to the symmetric non-positive matrix $A_{1} \kappa\left(\partial \kappa / \partial x_{1}\right)+\kappa \kappa^{+} A_{1}$ and we get

$$
\operatorname{Re}\left(\kappa\left(\partial \kappa / \partial x_{1}\right) A_{1} v, v\right) \leq-\operatorname{Re}\left(\kappa \kappa^{+} A_{1} v, v\right)+C_{6 \lambda}\|u\|_{t-\mu / 2}^{2},
$$


with $\mu<\sigma / 2$ if $1<\sigma<2$ and $\mu=1$ if $\sigma>2$ (see [1]). Then

(9)

$$
\begin{aligned}
\left|\left(\kappa \kappa^{+} A_{1} v, v\right)\right| & \leq\left|\left(A_{1} \kappa v, \kappa^{+} v\right)\right|+\left|\left(\left[A_{1}, \kappa\right] v, \kappa^{+} v\right)\right| \\
& \leq C_{7}\left(\|\kappa v\| \cdot\left\|\kappa^{+} v\right\|+\|v\|_{-1} \cdot\left\|\kappa^{+} v\right\|\right) \\
& \leq C_{7}\|Q u\| \cdot\left\|Q^{+} u\right\|+C_{7 \lambda}\|u\|_{t-1} \cdot\left\|Q^{+} u\right\|
\end{aligned}
$$

and $Q^{+}=Q_{\lambda, \delta_{1}}^{+}$is defined as $Q_{\lambda, \delta_{1}}$ with $\kappa$ replaced by $\kappa^{+}$. Note that $\left\|Q^{+} u\right\|<\infty$ as $Q^{+}(x, \xi)$ concentrates in a ngbhd of $\left\{x_{1}=b_{1}\right\} \times \Gamma_{\xi}$ and $u \in \mathcal{H}_{\text {mcl }}^{t}\left(\partial W \cap\left\{x_{1} \geq\right.\right.$ $\delta\}), \delta=a_{1}$.

By the identity $\partial Q / \partial x_{j}=e^{\lambda x_{1}}\left(\partial \kappa / \partial x_{j}\right)\left(1+\left|\delta_{1} \xi\right|^{2}\right)^{-1} h(\xi), j \geq 2, \partial Q / \partial x_{j}$ concentrates in a ngbhd of $\left\{x_{j}=a_{j}\right\} \times \Gamma_{\xi},\left\{x_{j}=b_{j}\right\} \times \Gamma_{\xi}$ and simple computations show that

$$
\begin{aligned}
\mid\left(\partial Q / \partial x_{j}(x, D) A_{j}\right. & (x) u, Q u) \mid \\
& \leq\left|\left(A_{j}\left(\partial Q / \partial x_{j}\right) u, Q u\right)\right|+\left|\left(\left[A_{j}, \partial Q / \partial x_{j}\right] u, Q u\right)\right| \\
& \leq C_{8 \lambda}\|u\|_{\mathcal{H}_{\mathrm{mcl}}^{t}\left(\partial W \cap\left\{x_{1} \geq \delta\right\}\right)}\|Q u\|_{0}+C_{9 \lambda}\|u\|_{t-1}\|Q u\|_{0} \\
& \leq\|Q u\|_{0}^{2}+C_{10 \lambda}\left(\|u\|_{t-1}^{2}+\|u\|_{\mathcal{H}_{\mathrm{mcl}}^{t}\left(\partial W \cap\left\{x_{1} \geq \delta\right\}\right)}\right) .
\end{aligned}
$$

Now we will estimate $\left(\left(\partial A_{j} / \partial x_{k}\right) D_{j}\left(\partial Q / \partial \xi_{k}\right)(x, D) u, Q u\right)$. To do this two terms will be considered, namely

$$
\begin{aligned}
& I I I_{1}=\left(\left(\partial A_{j} / \partial x_{k}\right) e^{\lambda x_{1}} \kappa(x) D_{j}\left(\partial h / \partial \xi_{k}\right)(D)\left(1+\left|\delta_{1} D\right|^{2}\right)^{-1} u, Q u\right), \\
& I I I_{2}=\left(\left(\partial A_{j} / \partial x_{k}\right) e^{\lambda x_{1}} \kappa(x) h(D) D_{j} \delta_{1}^{2} D_{k}\left(1+\left|\delta_{1} D\right|^{2}\right)^{-2} u, Q u\right) .
\end{aligned}
$$

Obviously, $\delta_{1}^{2} \xi_{k}\left(1+\delta_{1}^{2}|\xi|^{2}\right)^{-2}$ is uniformly bounded in $S_{1,0}^{-1,0}, \Sigma_{\varrho}^{-1}, \varrho>0, \varrho$ not an integer, $\forall \delta_{1} \in(0,1]$. The observations that $\delta_{1}^{2} \xi_{j} \xi_{k}\left(1+\left|\delta_{1} \xi\right|^{2}\right)^{-1}$ is uniformly bounded in $S_{1,0}^{0}$ with respect to $\delta_{1}>0$ and

$$
e^{\lambda x_{1}} \kappa(x) h(D) \delta_{1}^{2} D_{j} D_{k}\left(1+\left|\delta_{1} D\right|^{2}\right)^{-2} u=Q\left(\delta_{1}^{2} D_{j} D_{k}\left(1+\left|\delta_{1} D\right|^{2}\right)^{-1} u\right)
$$

enable us to conclude that

$$
\left|I I I_{2}\right| \leq C_{11}\|Q u\|_{0}^{2}+C_{11 \lambda}\|u\|_{t-1 / 2}^{2} .
$$

The cut-off symbol $h(\xi)$ can be written as $h(\xi)=|\xi|^{t} c(\xi), \operatorname{ord}_{\xi} c=0,0 \leq c \leq 1$, $c \equiv 1$ in a conic ngbhd of $\Gamma_{\xi}$ and $c \equiv 0$ outside a larger conic ngbhd of $\Gamma_{\xi}$. The inequality

$$
\left|\partial h / \partial \xi_{k}\right|^{2} \leq 2 t^{2}\left(h^{2} /|\xi|^{2}\right)+2|\xi|^{2 t}\left|\partial c / \partial \xi_{k}\right|^{2}
$$

will be useful later. Thus

$$
\begin{aligned}
& \left\|e^{\lambda x_{1}} \kappa D_{j}\left(\partial h / \partial \xi_{k}\right)(D)\left(1+\left|\delta_{1} D\right|^{2}\right)^{-1} u\right\|_{0} \\
& \quad \leq\left\|D_{j}\left(\partial h / \partial \xi_{k}\right)(D)\left(1+\left|\delta_{1} D\right|^{2}\right)^{-1}\left(e^{\lambda x_{1}} \kappa(x) u\right)\right\|_{0}+C_{12 \lambda}\|u\|_{t-1} .
\end{aligned}
$$


On the other hand, according to (12),

$$
\begin{aligned}
\| D_{j}\left(\partial h / \partial \xi_{k}\right)(D) & \left(1+\left|\delta_{1} D\right|^{2}\right)^{-1}\left(e^{\lambda x_{1}} \kappa(x) u\right) \|_{0}^{2} \\
= & \int \xi_{j}^{2}\left(\partial h / \partial \xi_{k}\right)^{2}\left(1+\left|\delta_{1} \xi\right|^{2}\right)^{-2}\left|\left(e^{\lambda x_{1}} \kappa(x) u\right)^{\wedge}\right|^{2}(\xi) d \xi \\
\leq & 2 t^{2}\left\|h(D)\left(1+\left|\delta_{1} D\right|^{2}\right)^{-1}\left(e^{\lambda x_{1}} \kappa u\right)\right\|_{0}^{2} \\
& +2 \int|\xi|^{2 t+2}\left(\partial c / \partial \xi_{k}\right)^{2}\left|\left(e^{\lambda x_{1}} \kappa(x) u\right)^{\wedge}\right|^{2}(\xi) d \xi \\
\leq & 2 t^{2}\|Q u\|_{0}^{2}+C_{13 \lambda}\|u\|_{t-1}^{2}+2\left\||D|^{t+1}\left(\partial c / \partial \xi_{k}\right)\left(e^{\lambda x_{1}} \kappa u\right)\right\|_{0}^{2} \\
\leq & \left.2 t^{2}\|Q u\|_{0}^{2}+C_{13 \lambda}\|u\|_{t-1}^{2}+C_{14 \lambda}\|u\|_{\mathcal{H}_{\mathrm{mcl}}^{t}\left(\partial W \cap\left\{x_{1} \geq \delta\right\}\right)}^{2}\right) .
\end{aligned}
$$

We remind the reader that $\operatorname{ord}_{\xi}|\xi|^{t+1}\left(\partial c / \partial \xi_{k}\right)=t$ and $\kappa(x)\left(\partial c / \partial \xi_{k}\right)$ concentrates in a conic ngbhd of $\Delta \times \partial \Gamma_{\xi}$. In other words,

$$
\left|I I I_{1}\right| \leq C_{15}\|Q u\|_{0}^{2}+C_{16 \lambda}\left(\|u\|_{t-1}^{2}+\|u\|_{\mathcal{H}_{\mathrm{mcl}}^{t}}^{2}\left(\partial W \cap\left\{x_{1} \geq \delta\right\}\right)\right) .
$$

Combining the identity (3) and the corresponding estimates (5) for $I$, (6)-(11), (13) for $I I$ and

$$
\operatorname{Im}(Q P u, Q u) \geq-2\|Q P u\|_{0}^{2}-2\|Q u\|_{0}^{2}
$$

we come to the conclusion that

$$
\begin{aligned}
& (\lambda-C)\|Q u\|_{0}^{2} \leq 2\|Q P u\|_{0}^{2}+C\left\|Q^{+} u\right\|_{0}^{2} \\
& \quad+K_{\lambda}\left(\|u\|_{t-1 / 2}^{2}+\|u\|_{t-\mu / 2}^{2}+\|u\|_{t+(1-\sigma) / 2}^{2}+\|u\|_{\mathcal{H}_{\text {mcl }}^{t}\left(\partial W \cap\left\{x_{1} \geq \delta\right\}\right)}^{2}\right) .
\end{aligned}
$$

The constant $C$ does not depend on $\lambda>0$ and $\delta_{1}>0$, and $K_{\lambda}$ depends on $\lambda>0$ only. Taking $\lambda$ sufficiently large and letting $\delta_{1} \rightarrow 0$ we prove Theorem 2 for $\sigma>2$.

To consider the case $1<\sigma<2$ we have to modify the proof of our Theorem 2 assuming $P u \in \mathcal{H}_{\text {mcl }}^{t}, u \in \mathcal{H}_{\text {mcl }}^{t}(\partial W)$ and $u \in \mathcal{H}_{\text {comp }}^{t-\gamma}(X), 0<\gamma<1 / 2$, instead of $\gamma=1 / 2$ etc.

\section{References}

[1] J. M. Bony, Calcul symbolique et propagation des singularités pour les équations aux dérivées partielles non-linéaires, Ann. Sci. Ecole Norm. Sup. (4) 14 (1981), 209-246.

[2] L. Hörmander, The Analysis of Linear Partial Differential Operators IV, Springer, Berlin 1985.

[3] V. I. Ivriŭ, Wave fronts of solutions of symmetric pseudodifferential systems, Sibirsk. Mat. Zh. 20 (1979), 557-578 (in Russian).

[4] P. Popivanov, Wave fronts of the solutions of some classes of non-linear partial differential equations, C. R. Acad. Bulgare Sci. 40 (11) (1987), 27-28. 